\title{
Conocimientos sobre atención primaria en salud de trabajadores de salud en Lempira y Francisco Morazán, 2010-2011
}

\author{
Emilia Lorenza Alduvín Sainz*
}

\section{RESUMEN}

Este estudio analiza los conocimientos en Participación Social, Intersectorialidad y Atención Familiar y Comunitaria, de la Estrategia de Atención Primaria en Salud APS que tienen los recursos humanos de las Unidades de Salud (US) con modelo de gestión centralizado y descentralizado de Lempira y Francisco Morazán, Honduras, durante 2010-2011. Es un estudio cuantitativo, transversal, comparativo, desarrollado en veintinueve municipios. Se seleccionaron US comparables, con más de un año en funcionamiento, $50 \%$ de personal permanente, y similar nivel de complejidad. Se aplicó un instrumento de conocimientos a 109 recursos humanos de US centralizadas, y a 118 de descentralizadas, entre Médicos, Licenciadas en Enfermería, Auxiliares de Enfermería y Bachilleres Técnicos en Salud. Los resultados indican que las US descentralizadas, dedican más personal para promoción de la salud; menos de la mitad de los entrevistados identifican valores y principios de APS, mayormente los Bachilleres Técnicos en Salud; no se evidenciaron diferencias significativas en conocimientos en APS entre ambos modelos de gestión, sino más bien, por profesiones. Se concibe APS como labor no profesional.

En Participación Comunitaria e Intersectorialidad, los conocimientos de los Bachilleres Técnicos en Salud fueron significativamente mayores. Se concluye que la implementación de la Estrategia APS continúa siendo un desafío en el sistema de salud en las regiones estudiadas; la autopercepción sobre los trabajadores de salud que aplican APS no guarda relación con el nivel de conocimientos en APS demostrado. Hace falta mayor desarrollo de equipos interdisciplinarios en el sistema de salud.

Palabras clave: Atención primaria en salud, recursos humanos, descentralización, intersectorialidad, participación

* Universidad Nacional Autónoma de Honduras. Facultad de Ciencias Médicas. Posgrado en Salud Pública. ealduvin@gmail.com 


\section{ABSTRACT}

This assessment analyzes knowledge on Social Participation, Intersectoral Collaboration and Family and Community Approach of the Primary Health Care Strategy PHC, held by human resources at Health Units $(\mathrm{HU})$ with a centralized and decentralized management models at Lempira and Francisco Morazán departments, Honduras, during 2010-2011. It is a quantitative, transversal, comparative study developed in twenty-nine municipalities. Inclusion criteria were comparability of $\mathrm{HUs}$ in terms of more than one year functioning, $50 \%$ of permanent staff, and similar complexity level. A knowledge based survey was applied to 109 staff members of centralized HU's, and to 118 of decentralized ones: Physicians, Professional Nurses, Auxiliary Nurses, and Health Technicians. Results pointed out that decentralized HU's dedicate more staff members to Health Promotion; less than $50 \%$ identify values and principles of PHC, mainly Health Technicians; significant differences about PHC knowledge among different management models were not identified; however differences were found by profession held by health workers. $\mathrm{PHC}$ is conceived as a non-professional task.

Knowledge about Community Participation and Intersectoral Collaboration were significantly greater among Health Technicians. It was concluded that implanting the PHC Strategy remains a challenge in the health system; self-perception about fulfilling PHC does not correlate with demonstrated PHC knowledge. Greater development of interdisciplinary teams in the health system is strongly needed.

Key words: Primary health care, human resources, decentralization, intersectoral collaboration, participation. 


\section{INTRODUCCIÓN}

En los últimos 30 años, desde la Declaración de Alma-Ata sobre Atención Primaria en Salud APS, se han registrado avances significativos en el estado de salud mundial; pero también se han multiplicado las inequidades en salud entre los países y al interior de los mismos. Esta situación ha demandado revisar las políticas de salud, en el contexto mundial actual; discutiéndose si los principios originales de la Declaración de Alma-Ata, son hoy día, una opción viable. Los debates que en las últimas dos décadas se han dado entre un enfoque de entrega de servicios selectivo o integral, y entre el enfoque comunitario y el institucional, ahora se están moviendo hacia la combinación de las fortalezas de ambos tipos de enfoques. La participación comunitaria y la intersectorialidad han demostrado ser los componentes más débiles de la APS. La participación en salud fue un principio clave en la Declaración de Alma-Ata, ya que promueve la autoconfianza personal y comunitaria en el planeamiento, organización y control de laAPS; y es esencial para obtener mejores resultados. Una participación comunitaria sólida es importante, no solo para asegurar la respuesta gubernamental hacia las necesidades de la sociedad, sino para proporcionar un espacio activo a la comunidad para el diseño, implementación y sostenibilidad de acciones integrales de los sistemas de salud. Parte importante de esta variación en el modelo de servicios es la descentralización; estrategia que en Honduras ha venido implementándose en algunos departamentos pioneros en el país, como Lempira y Francisco Morazán. La descentralización ha demandado del desarrollo de nuevas habilidades en los trabajadores de salud, como el fortalecimiento del trabajo comunitario, la participación y las acciones intersectoriales entre salud y desarrollo.

La integración de servicios, por su parte, puede ser mejor concebida como un continuo, más que como extremos integrado/no integrado. Involucra discusiones entre la organización de varias tareas, necesarias para proporcionar servicios de calidad, e involucra una mezcla de acciones políticas, técnicas y administrativas. Para el logro de dichos retos, se necesitan nuevas competencias para sustituir los modelos de gestión excesivamente simplistas del pasado por una mediación eficaz en salud que adopte nuevos mecanismos para el diálogo multilateral sobre políticas a fin de formular las orientaciones estratégicas de las reformas de la APS. Honduras, como el resto de países de Latinoamérica, se encuentra en una etapa de transición entre la declaratoria de principios y valores de laAPS y la implementación de la misma, superando el enfoque selectivo. Entender la APS como puerta de entrada al sistema sigue siendo un reto pendiente, ya que no se ha llegado a una conceptualización consensuada, ni a una institucionalización de los procesos 
necesarios para darle curso, debido a la fragmentación del sistema y a los múltiples intereses prevalecientes. ${ }^{7}$ La insuficiencia de médicos, enfermeras, odontólogos, y otro personal técnico, con las competencias requeridas es aún una de las razones impide el pleno desarrollo del potencial de la estrategia APS para la construcción de sistemas de salud más efectivos y equitativos. ${ }^{8}$ Este potencial, no obstante, debe de considerar también las características de las poblaciones en las cuales se desarrolla la estrategia. Para 2010, el departamento de Francisco Morazán contaba con 1, 433,810 habitantes, presentaba, un IDH 0.763, mayor que el promedio nacional; no obstante, la variabilidad del IDH en los seis municipios estudiados de Francisco Morazán, osciló entre 0.662 para Sabanagrande y 0.550 para Curarén. ${ }^{9}$ Según la ENDESA 2005-2006, la distribución de la riqueza en la población del área rural de Francisco Morazán oscilaba entre $26 \%$ en quintil inferior y $8.2 \%$ en quintil superior. ${ }^{10}$ Por su parte, el departamento de Lempira tenía para 2010 una población de 315,565 habitantes en sus 28 municipios, siendo su IDH promedio, de 0.592; y oscilando el IDH intermunicipal entre 0.647 en el municipio de Las Flores y 0.499 para San Andrés. La distribución de la riqueza en Lempira oscilaba, según últimos datos de ENDESA, entre $63.9 \%$ en el quintil más pobre y un $1.6 \%$ de la población en el quintil superior.

\section{METODOS YTÉCNICAS}

Se diseñó un estudio cuantitativo, transversal, co-relacional; que compara los conocimientos de los distintos tipos de trabajadores de salud laborando en unidades de salud en ambos modos de gestión, centralizados y descentralizados, en dos de los departamentos pioneros en la experiencia de descentralización de servicios de salud, que cuentan con varias unidades de salud con modelo de gestión centralizado y descentralizado, por lo que constituyeron lugares ideales para el estudio. Se eligieron unidades de salud con nivel de complejidad comparable, en 23 de los 28 municipios de Lempira, y en seis de los 28 municipios de Francisco Morazán. Las US centralizadas del departamento de Lempira se ubican en: Gracias, Erandique, San Francisco, Las Flores, San Rafael, San Francisco, Santa Cruz, Belén, La Campa, La Iguala, La Unión y Talgua; en tanto que las US descentralizadas, estudiadas se encuentran en: Guarita, Cololaca, Lepaera, San Manuel de Colohete, Gualcince, San Andrés, Piraera, La Virtud, Mapulaca, Virginia y Candelaria. En Francisco Morazán, las US centralizadas se ubican en los municipios de Sabanagrande, Reitoca, La Libertad y San Miguelito; en tanto que las US descentralizadas se ubican en Alubarén y Curarén. Todas las US cumplieron con los criterios de inclusión: más de un año de funcionamiento, $50 \%$ de trabajadores permanentes y nivel de complejidad, resolución y población adscrita similar. Se encuestó un total de 227 
trabajadores de salud: 31 médicos, que corresponden al 13.7\%; 19 enfermeras profesionales, correspondientes al 8.4\%; 138 auxiliares de enfermería, que representan el $60.8 \%$; 22 técnicos en salud ambiental, representando el $9.7 \%$ y 17 de otras profesiones. Los trabajadores pertenecen a las 79 unidades de salud seleccionadas; siendo estas, 45 Centros de Salud Rural CESAR (56.96\%); 31 Centros de Salud con Médico CESAMO (39.24\%), y 3 Clínicas Materno-Infantiles CMI (3.79\%). Se elaboró y validó un instrumento de conocimientos sobre APS, con énfasis en los principios y elementos de Abordaje Familiar y Comunitario, Participación Social e Intersectorialidad. Se elaboró una base de datos en SPSS, versión 19 donde se vació la información de las personas encuestadas. Se aplicaron técnicas estadísticas descriptivas y analíticas pertinentes: frecuencias, porcentajes y análisis de la varianza para evidenciar las diferencias en conocimientos entre los trabajadores de las US centralizadas y descentralizadas; por su formación profesional y antigüedad.

\section{ANÁLISIS}

\section{1) Caracterizaciones}

a) Caracterización de las Unidades de Salud. El $48 \%$ de las personas entrevistadas pertenecen a US con modo de gestión centralizado y el $52 \%$ laboran en US descentralizadas. El número de trabajadores de salud se reparten de manera proporcional; no obstante, las US descentralizadas destinan más personal a labores de promoción de la salud, como se observa en los siguientes gráficos:

\section{Gráfico No. 1. Número de recursos humanos en la Unidad de Salud.}

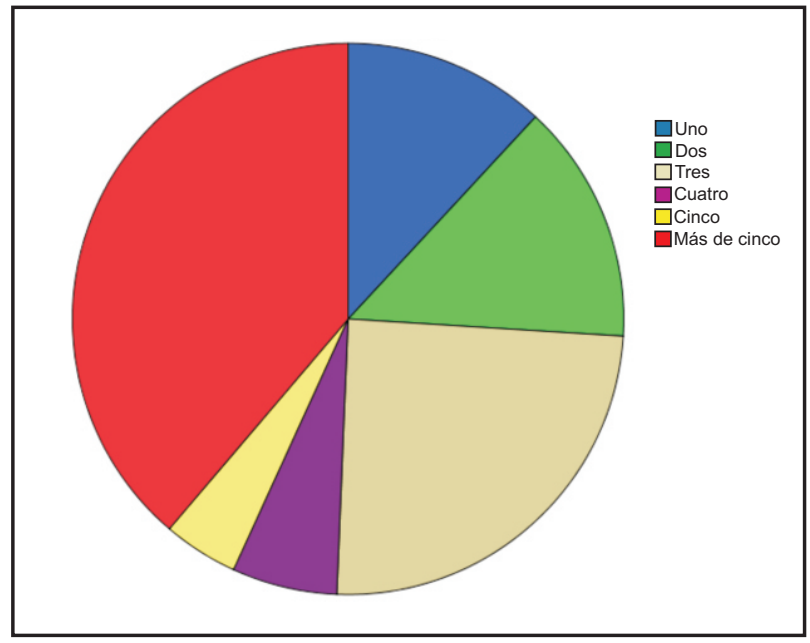




\section{Gráfico No. 2. Modo de gestión de la Unidad de Salud.}

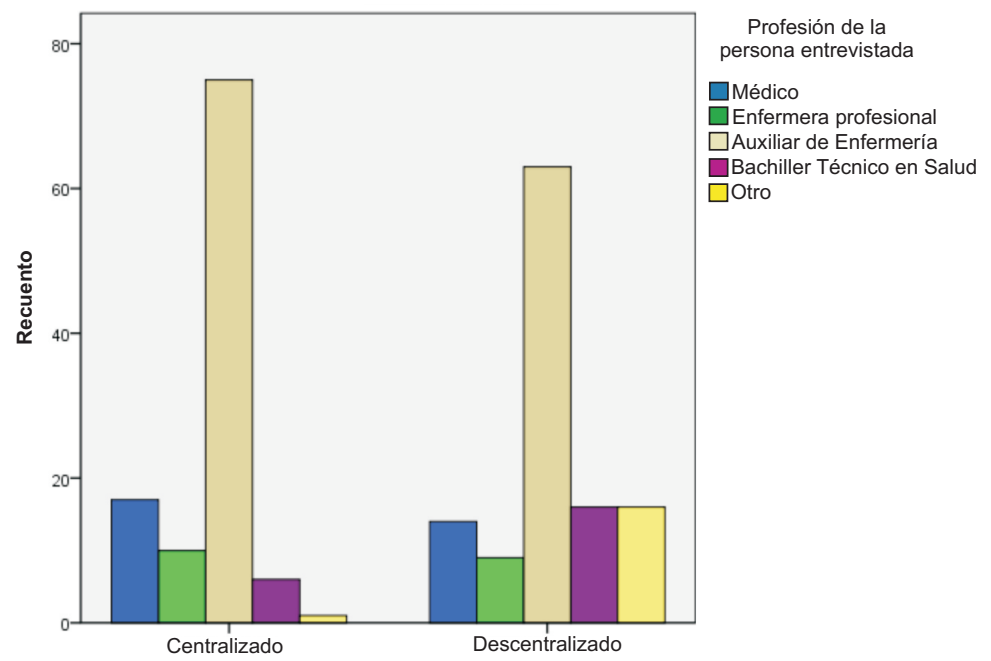

b) Caracterización de los trabajadores de salud entrevistados. Los trabajadores de salud entrevistados fueron en un $66.5 \%$ mujeres y un $33.5 \%$ hombres. La distribución por rangos de edad fue de $2.2 \%$ de menores de 20 años; $34.8 \%$ entre 20 y 29 años; $36.6 \%$ entre 30 y 39 años; un $18.1 \%$ entre 40 y 49 años; y el restante $8.4 \%$ de mayores de 50 años. En relación con la antigüedad de los trabajadores de salud en las US en que laboran al momento del estudio, la distribución fue de un $28.2 \%$ con menos de un año de laborar; un $28.6 \%$ de entre uno y tres años de laborar; el $10.1 \%$ entre 3 y 5 años de antigüedad; y un $33 \%$ con más de cinco años.

\section{Gráfico No. 3. Antiguedad de la persona entrevistada de trabajar en la Unidad de Salud.}

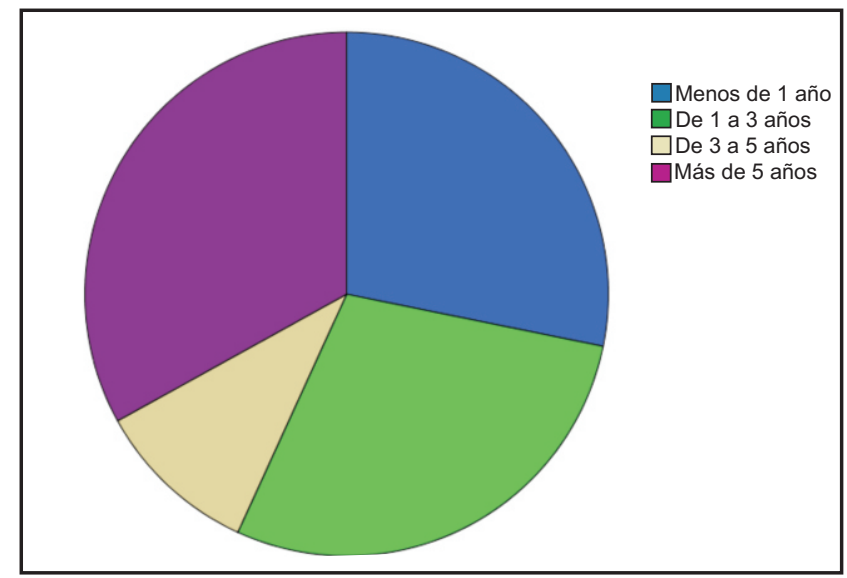


2) Conocimiento de los trabajadores de salud: a) Conocimiento sobre Atención Primaria en Salud. Un $40.5 \%$ de trabajadores no contestó o lo hizo de forma equivocada. El restante porcentaje (59.5\%) conceptualizó APS como: modelo de atención, estrategia de atención con valores y principios, provisión de "paquete básico de servicios", servicios para población rural, servicios para población de escasos recursos; y primer contacto de atención.

Sobre cuáles son los profesionales que aplican la APS en los servicios de salud, los trabajadores manifestaron, en orden de importancia, que son, primordialmente: promotores de salud, enfermeras auxiliares, médicos y enfermeras profesionales; en un segundo bloque de respuestas, aparecen, con un porcentaje mucho menor, los odontólogos, psicólogos y trabajadores sociales. El gráfico $\mathrm{N}^{\circ} 4$ muestra dichas variantes.

\section{Gráfico No. 4. Porcentaje de trabajadores que opinan cuales profesionales aplican APS.}

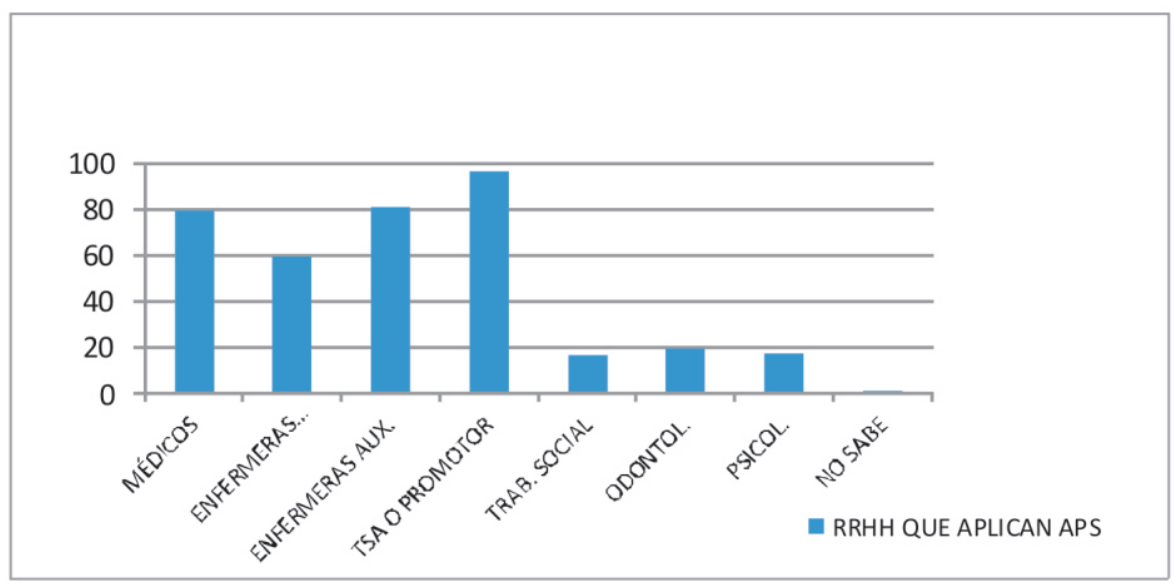

En relación con los principios (participación social, intersectorialidad, sostenibilidad, responsabilidad y rendición de cuentas, justicia social, respuesta a necesidades de la población) y valores (derecho al más alto nivel posible de salud, equidad y solidaridad) de la APS, no hubo respuestas o fueron equivocadas en un $85 \%$ para los principios y de $81.5 \%$ para los valores. b) Conocimientos sobre Atención Familiary Comunitaria.

El $72.7 \%$ de los entrevistados manifestaron no haber recibido capacitación sobre Atención Familiar y Comunitaria. A continuación los conocimientos sobre Condicionantes de salud mencionadas por los trabajadores de salud. 


\section{Cuadro No. 1. Condicionantes de salud del individuo mencionadas por trabajadores.}

\begin{tabular}{l|l}
\hline $\begin{array}{l}\text { Estilos de vida (ejercicio, no fumar, dieta balanceada, revisión médica } \\
\text { rutinaria) }\end{array}$ & $60.7 \%$ \\
\hline Condiciones socioeconómicas y culturales & $35.2 \%$ \\
\hline Factores ambientales (contaminación, smog) & $22.0 \%$ \\
\hline Factores biológicos y genéticos (edad, sexo, antecedentes familiares) & $10.8 \%$ \\
\hline $\begin{array}{l}\text { Acceso a servicios de salud } \\
\text { Condiciones de vivienda (piso de tierra, acceso a agua potable, número }\end{array}$ & $7.0 \%$ \\
\hline $\begin{array}{l}\text { de habitaciones) } \\
\text { Condiciones laborales (stress, desempleo, salario) }\end{array}$ & $1.7 \%$ \\
\hline
\end{tabular}

El tipo de respuestas emitidas por los trabajadores de salud nos sugiere una visión sobre las condicionantes de salud sobre las que, supuestamente, la población podría hacer mucho en función de sus estilos de vida, pero que, paradójicamente no es objeto de desarrollo de acciones de promoción de la salud de parte de los servicios de salud existentes, para ayudar a la población a alcanzar dichos objetivos.

Los conocimientos de los entrevistados sobre los criterios para la elaboración de un diagnóstico de familias en riesgo, que se muestran en el siguiente gráfico, sugieren una óptica más inclinada hacia criterios de tipo personal, que de los de tipo social.

\section{Gráfico No. 5. Criterios de familias en riesgo.}

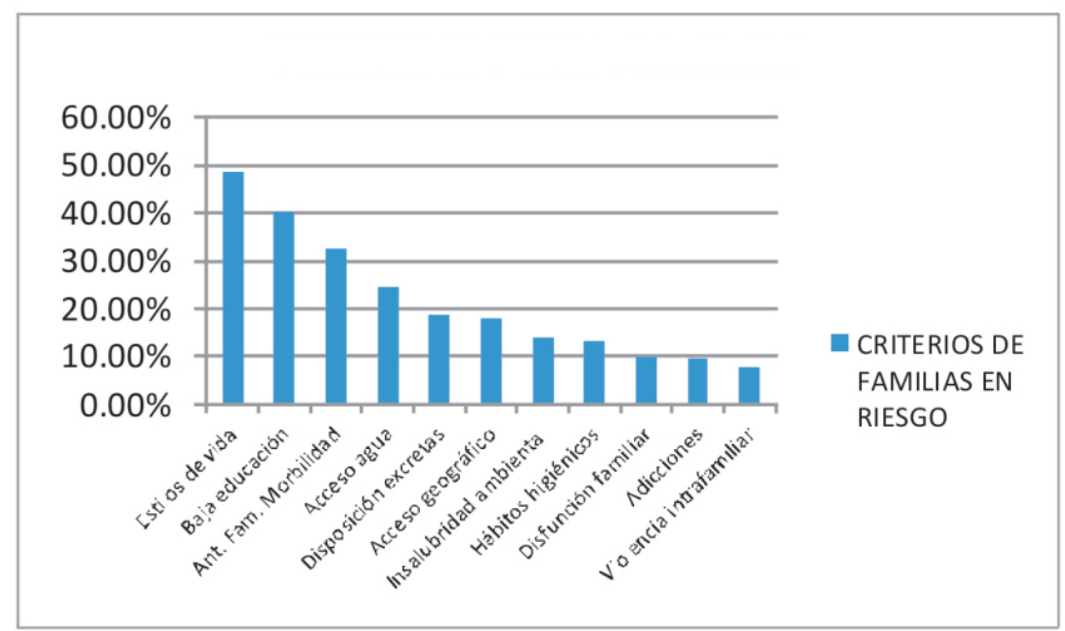

Los conocimientos sobre acciones comunitarias que se realizan con propósitos de promoción de la salud, que fueron manifestados por los entrevistados se presentan en el siguiente cuadro. 


\section{Cuadro No. 2. Mención de acciones y propósitos para la promoción de la salud.}

\begin{tabular}{l|c|l|c|}
\hline \multicolumn{1}{|c|}{ Sólo Acción } & $\%$ & \multicolumn{1}{c|}{ Acción más Propósito } & $\%$ \\
\hline Desarrollar campañas de promoción & 16.7 & $\begin{array}{l}\text {..para mejorar las condiciones de vida y } \\
\text { salud }\end{array}$ & 24.2 \\
\hline $\begin{array}{l}\text { Organizar comunidad: patronatos, juntas } \\
\text { Identificar y capacitar líderes }\end{array}$ & 10.6 & $\begin{array}{l}\text {..para empoderar y desarrollar capacidades } \\
\text {.para procurar sostenibilidad en los }\end{array}$ & 24.7 \\
\hline $\begin{array}{l}\text { Organizar grupos en espacios de } \\
\text { atención especial (diabetes, VIH, niñez) }\end{array}$ & 2.6 & $\begin{array}{l}\text {..para desarrollar acciones de cuidado, } \\
\text { solidaridad y apoyo }\end{array}$ & 4.8 \\
\hline
\end{tabular}

Los datos del cuadro anterior, nos muestran una tendencia de entendimiento muy limitada sobre la promoción de la salud y la organización comunitaria; ambas, piezas clave de la estrategia de APS. b) Conocimientos sobre participación comunitaria. A pesar de que un $81.1 \%$ de los trabajadores entrevistados manifestó no haber recibido capacitaciones sobre organización comunitaria; el 59.9\% mencionó componentes para una matriz de plan de acción comunitario, señalando mecanismos para identificar actores en la comunidad, en un $43.1 \%$, e identificando elementos para priorizar necesidades de salud de la población, en un $40.5 \%$. Algunos otros conocimientos relevantes sobre participación comunitaria, se muestran en el siguiente gráfico $\mathrm{N}^{\circ} 6$.

\section{Gráfico No. 6. Conocimientos para trabajo comunitario.}
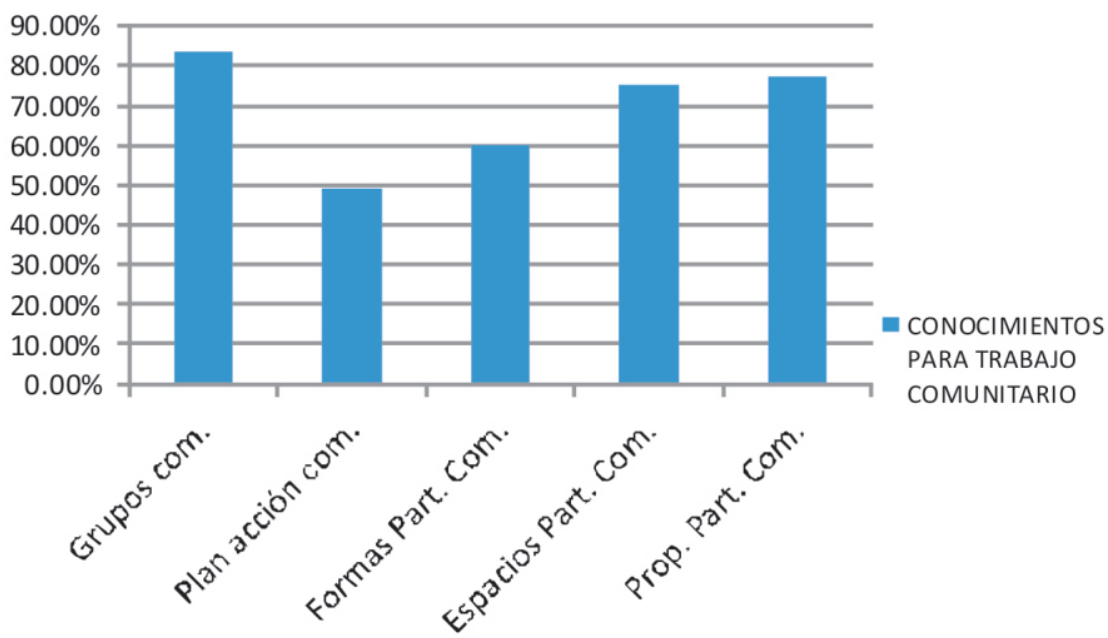
La información que nos muestra el gráfico anterior, indica que, si bien los trabajadores de salud tienen los conocimientos para identificar actores comunitarios y definir los propósitos de las reuniones en cerca del $80 \%$; la visión sobre su participación en aspectos concernientes a considerar para un plan de acción comunitario: priorizar problemas de manera conjunta, plantear propuestas de cambio, definir actividades concretas de participación de la población y establecer indicadores de evaluación, solamente se manifestó en un $49.3 \%$ de los entrevistados; así como con las formas de participación comunitaria: recoger la información y opinión de la comunidad; tomar decisiones conjuntas; dialogar, negociar y concertar; y pedir y rendir cuentas mutuamente; únicamente estuvo presente en un $59.9 \%$.

Estos resultados sugieren que los trabajadores de las US estudiadas, identifican a los miembros de la comunidad, pero no los consideran, ni aprovechan su potencial para involucrarles en la planificación y organización de actividades en salud. c) Conocimientos sobre intersectorialidad. Los conocimientos sobre intersectorialidad aparecen, en general, en mejores niveles que los conocimientos sobre participación comunitaria. Los ítems valorados fueron: identificación de actores comunitarios, 83.7\%; participantes en la elaboración de planes y proyectos de salud, $82.8 \%$; y mecanismos de comunicación efectiva entre los trabajadores de salud y la comunidad, 66.5\%, que comprende aspectos como: escuchar atentamente, tolerancia y apertura al diálogo, intercambio de ideas, divulgación de información y conocimientos, reparto de publicaciones e inclusión de personal bilingüe. Solo el $22 \%$ de los entrevistados ha recibido capacitación para formular proyectos.

\section{Gráfico No. 7. Conocimientos sobre intersectorialidad.}

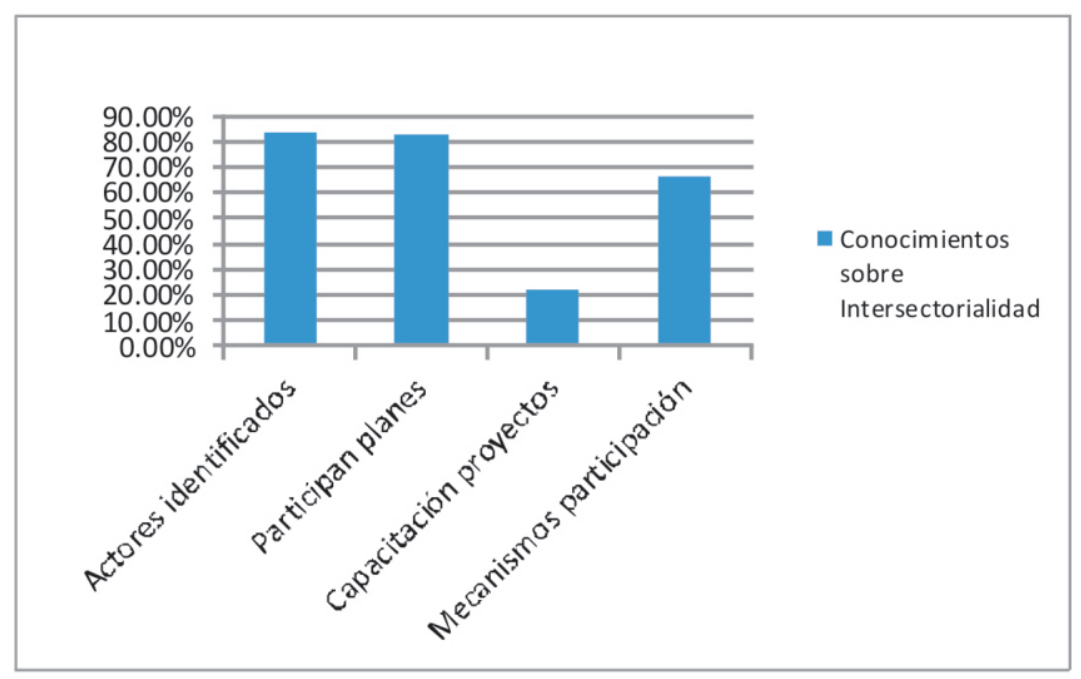


3) Diferencias identificadas. La hipótesis planteada en el estudio gira alrededor de las diferencias existentes en los conocimientos respecto de los componentes de APS Atención Familiar y Comunitaria, Participación Comunitaria e Intersectorialidad, que presentan los recursos humanos o trabajadores de salud de las US con modelo de gestión centralizado o descentralizado. Los resultados encontrados al analizar los datos, sugieren que no existen diferencias significativas en los conocimientos de los trabajadores de salud de ambos tipos de US. Al revisar la base de datos, se indagó también por posibles diferencias debidas a la antigüedad de los trabajadores en su espacio de trabajo. Respecto de esta variable no fueron encontradas diferencias significativas en los conocimientos sobre APS. La única variable que mostró un comportamiento significativamente diferente fue la de Profesión de la persona entrevistada: médicos, enfermeras profesionales, auxiliares de enfermería, bachilleres técnicos en salud. Los conocimientos identificados como significativamente diferentes por la profesión fueron 18 en total, abarcando los cuatro ámbitos de conocimientos explorados: Atención Primaria en Salud, Atención Familiar y Comunitaria, Participación Comunitaria e Intersectorialidad.

Las variables que tuvieron una diferencia significativa en el ámbito de Atención Primaria en Salud fueron: 1) Conocimiento sobre sí las enfermeras (os) aplican APS; 2) Conocimiento sobre sí los odontólogos (as) aplican APS; y 3) conocimientos sobre los principios de la APS. Con relación a los conocimientos sobre Atención Familiar y Comunitaria, las diferencias identificadas fueron en: 4) Estilos de vida que inciden en la salud; 5) Factores genéticos y biológicos que inciden en la salud; 6) Involucrar a las familias en actividades de salud para mejorar la participación y apropiación; 7) Incentivar el autocuidado y la autoestima para mejorar los estilos de vida saludables; 8) Desarrollar planes de intervención para disminuir las Necesidades Básicas Insatisfechas; 9) Organizar a la comunidad para empoderarlos y desarrollar sus capacidades; 10) Desarrollar campañas de promoción y prevención para mejorar las condiciones de vida y salud.

En relación con los conocimientos sobre Participación Comunitaria, se encontraron diferencias en los conocimientos respecto a: 11) Mecanismos para identificar actores comunitarios; 12) Elementos para priorizar necesidades de salud de la población; 13) Grupos u organizaciones que conforman un Comité de Salud; 14) Actividades para promover la participación comunitaria; y 15) Espacios de concertación con la comunidad. Por último, en el elemento de Intersectorialidad, se identificaron diferencias significativas sobre: 16) Conocimiento de actores a identificar en una comunidad; 17) Actores sociales participantes en la elaboración de planes y proyectos en salud; y 18) Mecanismos de comunicación efectiva. El 
siguiente cuadro muestra los valores para cada una de las variables sobre Conocimientos sobre APS; tipo de profesional que mejores conocimientos mostró en cada caso; y nivel de significancia de la diferencia encontrada respecto del resto de trabajadores.

\section{Cuadro No. 3. Conocimientos de APS significativamente distintos por tipo de profesión.}

\begin{tabular}{|c|c|c|c|c|c|}
\hline \multirow{2}{*}{$\begin{array}{c}\text { Conocimiento sobre elementos } \\
\text { de APS }\end{array}$} & \multicolumn{4}{|c|}{$\begin{array}{l}\text { \% Competencia } \\
\text { del Profesional }\end{array}$} & \multirow{2}{*}{$\begin{array}{c}\text { Significancia } \\
\text { de la } \\
\text { diferencia }\end{array}$} \\
\hline & 1 & 2 & 3 & 4 & \\
\hline Elementos de priorización de necesidades de salud & 32 & 47 & 39 & 73 & .000 \\
\hline Mención de espacios de concertación comunitaria & 77 & 74 & 74 & 95 & .000 \\
\hline Actividades para incentivar participación comunitaria & 45 & 42 & 46 & 86 & .000 \\
\hline Actores sociales a identificar en la comunidad & 97 & 89 & 80 & 90 & .001 \\
\hline Organización de grupos para desarrollar sus capacidades & 35 & 58 & 30 & 64 & .001 \\
\hline Determinante de salud: Factores genéticos y biológicos & 32 & 11 & 8 & 0 & .002 \\
\hline Planes para disminuir necesidades básicas insatisfechas & 26 & 16 & 21 & 50 & .003 \\
\hline Actores para elaboración de planes y proyectos & 97 & 79 & 80 & 90 & .007 \\
\hline $\begin{array}{l}\text { Mención de mecanismos para identificación de actores } \\
\text { comunitarios }\end{array}$ & 45 & 47 & 40 & 73 & .008 \\
\hline Determinante de salud: Estilos de vida & 52 & 53 & 64 & 73 & .009 \\
\hline Mención de mecanismos de comunicación efectiva & 64 & 79 & 62 & 86 & .013 \\
\hline $\begin{array}{l}\text { Involucrar a la comunidad para apropiación de acciones en } \\
\text { salud }\end{array}$ & 23 & 22 & 22 & 50 & .015 \\
\hline $\begin{array}{l}\text { identificación de grupos en la comunidad para comité de } \\
\text { salud }\end{array}$ & 71 & 84 & 79 & 41 & .017 \\
\hline Actividades de promoción para mejorar condiciones de vida & 42 & 37 & 43 & 54 & .018 \\
\hline Mención de los principios de la APS & 16 & 11 & 12 & 27 & .018 \\
\hline $\begin{array}{l}\text { Incentivar autocuidado y autoestima para mejorar los estilos } \\
\text { de vida }\end{array}$ & 48 & 42 & 44 & 73 & .020 \\
\hline Enfermeras (os) aplican APS & 67 & 84 & 57 & 36 & .025 \\
\hline Odontólogos (as) aplican APS & 13 & 32 & 19 & 9 & .057 \\
\hline
\end{tabular}

Al analizar algunos resultados del cuadro anterior, podemos advertir que en primer lugar resaltan los altos porcentajes sobre conocimientos de APS que de manera consistente mostraron los Bachilleres Técnicos en Salud, es decir, trabajadores de salud no universitarios. Estos mayores conocimientos se revelan en diferentes áreas relacionadas la mayoría con actividades de participación comunitaria; no obstante, sus puntajes son significativamente mayores también en conocimientos conceptuales sobre APS. En segundo lugar sobre los conocimientos demostrados en APS, se ubican las Enfermeras Profesionales, pero en un nivel mucho menor que los Bachilleres Técnicos en Salud. Estos resultados contrastan abiertamente con la autopercepción que sobre su propio accionar en actividades de APS, guardan los

\footnotetext{
${ }^{1}$ Médicos

${ }^{2}$ Enfermeras Profesionales

${ }^{3}$ Auxiliares de Enfermería

${ }^{4}$ Bachilleres Técnicos en Salud
} 
diferentes tipos de trabajadores de salud; y también sobre el conocimiento sobre los Principios de APS que tienen los entrevistados, como apreciamos en el siguiente cuadro.

\section{Cuadro No. 4. Comparación entre autopercepción sobre la aplicación de APS y conocimientos sobre los principios de APS según tipo de trabajadores de salud.}

\begin{tabular}{l|c|c|}
\hline $\begin{array}{c}\text { Tipo de trabajadores } \\
\text { de salud }\end{array}$ & $\begin{array}{c}\text { Autopercepcion sobre la } \\
\text { aplicación de APS }\end{array}$ & $\begin{array}{c}\text { Conocimiento sobre } \\
\text { principios de APS }\end{array}$ \\
\hline Médicos & $90 \%$ & $16 \%$ \\
\hline Enfermeras & $84 \%$ & $11 \%$ \\
\hline Auxiliares de Enfermería & $80 \%$ & $12 \%$ \\
\hline Técnicos en Salud Ambiental & $45 \%$ & $27 \%$ \\
\hline
\end{tabular}

Al analizar el cuadro anterior, a la luz de los datos del cuadro $N^{\circ} 3$, podemos deducir que la autopercepción sobre la aplicación de APS, y los conocimientos sobre principios APS, sugieren que existe un entendimiento diferente sobre la Atención Primaria en Salud entre los técnicos en salud ambiental, respecto de los médicos, auxiliares de enfermería y, en menor grado, enfermeras profesionales, lo cual pudiera estar teniendo una incidencia directa en limitar las actividades de programación y realización de acciones encaminadas al Abordaje Familiar y Comunitario; impidiendo el desarrollo de la Participación Comunitaria; y desaprovechando ventajas del trabajo Intersectorial.

\section{CONCLUSIONES}

Se concluyó que dos tercios de la población estudiada, ni conocen, ni se sienten responsables de aplicar los principios de Atención Primaria en Salud. El elemento mayormente desconocido de la APS se refiere al del Abordaje Familiar $y$ Comunitario, en lo que concierne a los conocimientos necesarios para la realización del diagnóstico de salud familiar; ésta situación, por tanto, les dificulta las posibilidades de identificar familias y comunidades en riesgo, elementos fundamentales para un abordaje desde la óptica de Atención Primaria en Salud. Tanto este aspecto, como los conocimientos sobre los principios de Participación Social e Intersectorialidad, son un desafío pendiente en las unidades de salud estudiadas de los departamentos de Lempira y Francisco Morazán, independientemente del modelo de gestión, sea centralizado o descentralizado, debido a que los resultados no mostraron diferencias significativas entre ambas subpoblaciones. Las únicas diferencias significativas encontradas en el presente 
estudio sobre los conocimientos de APS, se relacionaron con la variable Profesión de la persona entrevistada; encontrándose que los Técnicos en Salud Ambiental, seguidos de las Enfermeras Profesionales, son quienes mayor conocimiento sobre APS tienen, sobre todo en el terreno práctico; es decir, saben qué es lo que hay que haceren un modelo de salud orientado porAPS.

En contraste, estos conocimientos, no son manifestados por profesionales de la medicina, a pesar de que paradójicamente existe entre este gremio, la mayor autopercepción sobre quienes aplican APS en las unidades de salud. Dicho con otras palabras, el gremio médico manifiesta que aplican APS y, al mismo tiempo, reportan conocimientos significativamente limitados sobre el tema. Para finalizar, consideramos importante señalar que esta combinación de resultados, permite inferir implicaciones directas acerca del nivel de eficiencia que pudieran estar teniendo las labores relacionadas con APS en las US estudiadas; y sugiere la necesidad de realizar estudios que apunten a documentar la costo-efectividad de dichas labores, según el tipo de recurso humano que las realice.

Sin que sea precisamente el objetivo principal del presente estudio, pudo constatarse la ausencia de equipos multidisciplinarios en la estructura de las US estudiadas en los departamentos de Lempira y Francisco Morazán; equipos indispensables para la correcta implementación de la estrategia de APS, según se ha documentado exhaustivamente en la literatura sobre el tema.

\section{AGRADECIMIENTOS}

La autora desea expresar su sincero agradecimiento, a la Mtra. Mayra Isabel Discua Canales, a la Dra. Glady Paz y a la Lic. Iris Yolanda Díaz graduadas y egresada, respectivamente, de la Maestría en Salud Pública, por su colaboración en el levantamiento de la información y en la fase de análisis del presente estudio. Un agradecimiento especial a la Mtra. Astarté Alegría, que durante su período como Coordinadora General del Postgrado en Salud Pública, brindó un amplio respaldo y acompañamiento durante todas las fases del presente estudio. Se agradece a la Dirección de Investigación Científica, por el apoyo financiero obtenido a través de la Beca Básica de Investigación proporcionada a la autora, especialmente al Dr. Edwin Medina y la Lic. Margarita Arriaga, por las orientaciones recibidas para el manejo administrativo en la compra del equipo necesario para su realización y para el financiamiento parcial de las giras de campo necesarias para la aplicación de los instrumentos en los municipios estudiados. 


\section{BIBLIOGRAFÍA}

Harzeim, E., Casado, V. V., Bonal, P. P. (2009) La formación de profesionales de salud para la APS y Salud Familiar y Comunitaria en América Latina y Europa. EUROsociAL. Institut de recherche pour le dévelopment. Fundación para la Cooperación y Salud Internacional del Sistema Nacional de Salud de España. FIOCRUZ. Escuela Nacional de Salud Pública "Sergio Arouca". Río de Janeiro.

Lawn, J. E., Rifkins, S., Were, M., Vinod, K. P. \& Chopra, M. "Alma-Ata Rebirth and Revision \# 1. Alma-Ata 30 years on: revolutionary, relevant and time to revitalize". Lancet. Vol. 372 Sept 13, 2008. pp. 917-927.

Organización Mundial de la Salud. (1978) Declaración de Alma-Ata. Conferencia Internacional sobre Atención Primaria de Salud. Alma-Ata, URSS, 6 al 12 de Septiembre.

People's Health Movement, Health Action International, Médicos Internacionales \& Third World Network (2011) Global Health Watch 3. An alternative World Health Report. Zed Books. London|New York.

Programa de las Naciones Unidas para el Desarrollo (2011) Informe sobre Desarrollo Humano Honduras 2011. Reducir la inequidad: un desafío Impostergable PNUD. Tegucigalpa.

República de Honduras (2009) Atención Primaria en Salud: Puerta de entrada al Sistema de Salud de Honduras. Posibilidades y límites.Secretaría de Salud. Tegucigalpa.

República de Honduras. (2006) Encuesta Nacional de Demografía y Salud ENDESA 2005-2006. Instituto Nacional de Estadística. Tegucigalpa.

Rosato, M, et. al. "Alma-Ata Rebirth and Revision \# 5. Community participation: lessons from maternal, newborn and child health." Lancet. Vol. 372 Sept 13, 2008. pp. 962-971.

Velásquez, A. (2009) "Competencias necesarias de los recursos humanos para implementar las reformas del sistema de salud con base en la Atención Primaria de Salud" Reforma del Sector Salud. Lima.

Walley, J., et. al. "Alma-Ata Rebirth and Revision \# 8. Primary health care: making Alma-Ata reality." Lancet. Vol. 372 Sept 13, 2008. Pp. 1001-1007. 